\title{
Prevalence of Congenital Disorders of Glycosylation in Childhood Epilepsy and Effects of Anti-Epileptic Drugs on the Transferrin Isoelectric Focusing Test
}

\author{
Grace Silver ${ }^{1}$, Shalini Bahl ${ }^{1}$, Dawn Cordeiro ${ }^{1}$, Abhinav Thakral ${ }^{1}$, Taryn Athey ${ }^{2}$ \\ and Saadet Mercimek-Andrews 1,2,*(D) \\ 1 Department of Pediatrics, Division of Clinical and Metabolic Genetics, The Hospital for Sick Children, \\ Toronto, ON M5G 1X8, Canada; grace.silver@sickkids.ca (G.S.); shal.bahl@mail.utoronto.ca (S.B.); \\ dawn.cordeiro@sickkids.ca (D.C.); abhinav.thakral@mail.utoronto.ca (A.T.) \\ 2 Department of Medical Genetics, University of Alberta, Stollery Children's Hospital, \\ Edmonton, AB T6G 2H7, Canada; Taryn.Athey@albertahealthservices.ca \\ * Correspondence: saadet@ualberta.ca; Tel.: +1-780-492-0874
}

\section{check for}

updates

Citation: Silver, G.; Bahl, S.; Cordeiro, D.; Thakral, A.; Athey, T.; Mercimek-Andrews, S. Prevalence of Congenital Disorders of

Glycosylation in Childhood Epilepsy and Effects of Anti-Epileptic Drugs on the Transferrin Isoelectric Focusing Test. Genes 2021, 12, 1227. https://doi.org/10.3390/genes 12081227

Academic Editor: Bryan Mowry

Received: 8 May 2021

Accepted: 6 August 2021

Published: 10 August 2021

Publisher's Note: MDPI stays neutral with regard to jurisdictional claims in published maps and institutional affiliations.

Copyright: (c) 2021 by the authors. Licensee MDPI, Basel, Switzerland. This article is an open access article distributed under the terms and conditions of the Creative Commons Attribution (CC BY) license (https:/ / creativecommons.org/licenses/by/ $4.0 /)$.
Abstract: Introduction: Childhood epilepsy is one of the most common neurological problems. The transferrin isoelectric focusing (TIEF) test is a screening test for congenital disorders of glycosylation (CDG). We identified abnormal TIEF test in children with epilepsy in our epilepsy genetics clinic. To determine if an abnormal TIEF test is associated with anti-epileptic medications or abnormal liver functions, we performed a retrospective cohort study. Methods: This study was performed between January 2012 and March 2020. Electronic patient charts were reviewed. Standard non-parametric statistical tests were applied using R statistical software. Fischer's exact test was used for comparisons. Results: There were 206 patients. The TIEF test was abnormal in 11\% (23 out of 206) of the patients. Nine patients were diagnosed with CDG: PMM2-CDG $(n=5)$, ALG3-CDG $(n=1)$, ALG11-CDG $(n=2)$, SLC35A2-CDG $(n=1)$. We report 51 different genetic diseases in 84 patients. Two groups, (1) abnormal TIEF test; (2) normal TIEF test, showed statistically significant differences for abnormal liver functions and for valproic acid treatment. Conclusion: The TIEF test guided CDG diagnosis in $2.9 \%$ of the patients. Due to the high prevalence of CDG $(4.4 \%)$ in childhood epilepsy, the TIEF test might be included into the diagnostic investigations to allow earlier and cost-effective diagnosis.

Keywords: epilepsy; seizures; global developmental delay; congenital disorders of glycosylation; transferrin isoelectric focusing

\section{Introduction}

Childhood epilepsy is one of the most common neurological problems. The origin of epilepsy is known in about one-third of the children. There are numerous diagnostic investigations for childhood epilepsy. Some of these investigations are performed in pediatric, genetic, and neurology clinics to investigate the underlying etiologies for appropriate management. Metabolic investigations include plasma amino acids, acylcarnitine profile, total and free carnitines, homocysteine, transferrin isoelectric focusing (TIEF) tests, urine organic acids, and urine creatine and guanidinoacetate that may identify some of the treatable inherited metabolic disorders. Genetic investigations include microarray, a targeted next generation sequencing panel (TNGSP) for epilepsy, and exome sequencing (ES).

We previously reported the diagnostic yield of metabolic investigations (7\%) in childhood epilepsy [1]. The diagnostic yield of TNGSP for epilepsy was 19\%, and the diagnostic yield of ES was 37\% in our recent study [2].

The TIEF test is applied as a screening test for congenital disorders of glycosylation (CDG) in patients with global developmental delay and epilepsy in our epilepsy genetics clinic. Glycosylation is a process where sugar groups are made, modified and added onto 
proteins. Several rare, genetic metabolic disorders produce defects in glycosylation, with CDG being an umbrella term for these inherited metabolic disorders. The first CDG was reported in 1984 by Jaeken et al. [3]. Since then, more than 130 different genetic defects were reported under the category of CDG. Due to the genetic heterogeneity of CDG, there are numerous phenotypes associated with specific genotypes. Most patients present with multisystemic disease with a significant neurological component, often in the form of developmental delay. The TIEF test, which measures transferrin isoforms as a screening test of CDG type I (CDG-I) and type II (CDG-II), has been used for more than 25 years. We recently reported six different types of CDG in 15 patients from our center. About $50 \%$ of those patients presented with seizures or epilepsy [4].

We identified an abnormal TIEF test in children with epilepsy in our epilepsy genetics clinic who were seen for diagnostic investigations. We performed a retrospective cohort study to determine: (1) if an abnormal TIEF test is associated with a specific seizure type; (2) if an abnormal TIEF test is associated with certain anti-epileptic medications; (3) if an abnormal TIEF test is associated with abnormal liver functions; and (4) if an abnormal TIEF test is associated with any type of genetic disorder. We reviewed the literature for associations between anti-epileptic medications, abnormal liver functions, and abnormal TIEF tests.

\section{Materials and Methods}

This retrospective cohort study was performed in a single pediatric epilepsy genetics clinic at an academic health center between January 2012 and March 2020. Inclusion criteria were: (1) non-syndromic epilepsy; (2) seen in this epilepsy genetics clinic for diagnostic investigations; (3) underwent TIEF testing. Exclusion criteria were patients who had no TIEF test AND patients who had no history of epilepsy or seizure. The Institutional Research Ethics Board at The Hospital for Sick Children approved the study (Approval \#1000070163). Electronic patient charts were reviewed for clinical features, anti-epileptic medications, biochemical investigations, electrophysiological investigations, neuroimaging, and molecular genetic investigations including TNGSP, and ES. Information was entered into an Excel database (Microsoft Corp., Redmond, WA, USA). Liver function tests including AST, ALT, GGT, ALP, LDH, bilirubin, PT, and PTT, which were performed within one month of the TIEF test, were recorded. All anti-epileptic medications prior to and taken around the day(s) of the TIEF test were recorded. Patients' and parents' DNA samples were used for molecular genetic investigations according to the methods of clinical molecular genetic laboratories. The Alamut database was used for variant annotation for amino acid sequences, predictions of pathogenicity, and cross-species conservation of nucleotides. ACMG guidelines for the interpretation of variants were applied [5]. The Genome Aggregation Database was searched (gnomAD) (http:/ /gnomad.broadinstitute. org/about, accessed between February-May 2021) for the allele frequency of variants in the general population [6].

Standard non-parametric statistical tests were used for within-group comparisons. All analyses were performed using R statistical software. Fischer's exact test was used for comparisons. A two-tailed $p$ value $<0.05$ was considered statistically significant.

\section{Results}

There were 206 patients with childhood epilepsy fulfilling the inclusion criteria. All patients with abnormal TIEF tests are summarized for their clinical features, seizure history, anti-epileptic medications, liver function tests, and TIEF tests (patient and parents) in Table 1. We depicted the number of patients with normal and abnormal TIEF tests, their genetic diagnosis and type of molecular genetic investigation in Figure 1. 
Table 1. All patients with abnormal transferrin isoelectric focusing tests, their clinical features, liver functions, and transferrin isoelectric focusing results are summarized.

\begin{tabular}{|c|c|c|c|c|c|c|c|c|}
\hline $\begin{array}{l}\text { Patient Number/ Study } \\
\text { ID/Sex/Current Age } \\
\text { (Reference) }\end{array}$ & $\begin{array}{l}\text { Diagnosis (Genetic or } \\
\text { None) (Age of Diagnosis) }\end{array}$ & $\begin{array}{c}\text { Seizure Age of } \\
\text { Onset/Seizure Types }\end{array}$ & $\begin{array}{l}\text { Other Clinical } \\
\text { Features }\end{array}$ & $\begin{array}{l}\text { Anti-Epileptic } \\
\text { Medications Used }\end{array}$ & $\begin{array}{c}\text { Anti-Epileptic } \\
\text { Medications at the } \\
\text { Time of TIEF Test }\end{array}$ & $\begin{array}{c}\text { Liver Functions } \\
\text { AST/ALT/INR/GGT/ } \\
\text { ALP/Direct Bil }\end{array}$ & TIEF Test & Parental TIEI \\
\hline 1/015/F/2 yr(s) & $\begin{array}{l}S L C 35 A 2-\mathrm{CDG}(8 \mathrm{mo}(\mathrm{s})) \\
\text { by ES }\end{array}$ & $11 \mathrm{mo}(\mathrm{s}) / \mathrm{IS}$ & $\begin{array}{l}\text { GDD, FTT, dysmorphic } \\
\text { features (hypertelorism, } \\
\text { low set posteriorly } \\
\text { rotated ears, prominent } \\
\text { forehead, upslanting } \\
\text { palpebral fissures, short } \\
\text { nose with upturned } \\
\text { nose tip) }\end{array}$ & TPM, PRED, VGB & None & $\uparrow / \mathrm{N} / \mathrm{NA} / \mathrm{N} / \mathrm{NA} / \mathrm{NA}$ & $\begin{array}{c}\text { 个asialo, mono, di, tri } \\
\downarrow \text { tetra, penta }\end{array}$ & NA \\
\hline 2/031/F/10 yr(s) [1] & $\begin{array}{c}\text { ALG11-CDG (5 yr(s)) by } \\
\text { TNGSP for CDG (37 } \\
\text { genes) }\end{array}$ & $2 \mathrm{mo}(\mathrm{s}) / \mathrm{GTCS}, \mathrm{GTS}, \mathrm{IS}$ & $\begin{array}{c}\text { GDD, dystonia, } \\
\text { microcephaly, } \\
\text { dysmorphic features } \\
\text { (mild frontal bossing, } \\
\text { broad and tubular nose } \\
\text { with new onset of milia, } \\
\text { retrognathia, small } \\
\text { down-turned mouth, } \\
\text { chubby cheeks) }\end{array}$ & $\begin{array}{l}\text { VGB, PRED, ACTH, } \\
\text { TPM, LVT, CLB, LOR }\end{array}$ & VGB & $\mathrm{N} / \mathrm{N} / \mathrm{NA} / \mathrm{N} / \downarrow / \mathrm{N}$ & $\uparrow$ disialo & NA \\
\hline $3 / 215 / \mathrm{F} / 12 \mathrm{yr}(\mathrm{s})[1]$ & $\begin{array}{c}\text { ALG3-CDG (2 yr(s)) by } \\
\text { TIEF \& DS }\end{array}$ & Day 20/GTCS, GTS & $\begin{array}{c}\text { GDD, ataxia, spasticity, } \\
\text { dysmorphic features } \\
\text { (plagiocephaly, } \\
\text { micrognathia, tubular } \\
\text { nose) }\end{array}$ & PB & PB & $\mathrm{N} / \mathrm{N} / \mathrm{N} / \mathrm{N} / \mathrm{NA} / \mathrm{N}$ & $\uparrow$ asialo, $\uparrow$ disialo & NA \\
\hline 5/211/M/18 yr(s) & $\begin{array}{c}P M M 2-\mathrm{CDG}(16 \mathrm{yr}(\mathrm{s})) \text { by } \\
\text { ES }\end{array}$ & $\begin{array}{c}3.5 \mathrm{yr}(\mathrm{s}) / \mathrm{GTCS}, \mathrm{MS}, \\
\text { CPS }\end{array}$ & GDD, ataxia & $\mathrm{CBZ}, \mathrm{OXC}$ & None & N/N/N/N/NA/NA & $\begin{array}{c}\text { 个asialo, disialo } \\
\downarrow \text { tetra }\end{array}$ & NA \\
\hline $6 / 222 / F / 7$ yr(s) [1] & $\begin{array}{c}\text { PMM2-CDG (15 mo(s)) by } \\
\text { TNGSP for CDG (67 } \\
\text { genes) }\end{array}$ & $\begin{array}{l}2 \text { mo(s)/GTCS, GTS, } \\
\text { focal }\end{array}$ & $\begin{array}{l}\text { GDD, respiratory } \\
\text { distress, cardiac } \\
\text { abnormalities }\end{array}$ & $\mathrm{PB}, \mathrm{LOR}$ & None & N/NA/NA/NA/N/N & $\begin{array}{c}\uparrow \text { disialo } \\
\downarrow \text { trisialo, } \downarrow \text { tetrasialo }\end{array}$ & NA \\
\hline $7 / 224 / \mathrm{F} / 4 \mathrm{yr}(\mathrm{s})[1]$ & $\begin{array}{l}\text { PMM2-CDG (4 mo(s)) by } \\
\text { TIEF \& DS }\end{array}$ & Day 12/GTS & GDD, FTT & None & None & $\uparrow / \uparrow / N / N A / N A / N A$ & $\begin{array}{c}\uparrow \text { disialo } \\
\downarrow \text { tetrasialo }\end{array}$ & NA \\
\hline $8 / 230 / \mathrm{M} / 3 \mathrm{yr}(\mathrm{s})$ & $\begin{array}{c}\text { PMM2-CDG (3 yr(s)) by } \\
\text { TIEF \& DS }\end{array}$ & $18 \mathrm{mo}(\mathrm{s}) / \mathrm{GTCS}$ & $\begin{array}{l}\text { GDD, FTT, spasticity, } \\
\text { microcephaly, } \\
\text { dysmorphic features } \\
\text { (inverted nipples, } \\
\text { low-set ears) }\end{array}$ & None & None & $\uparrow / \uparrow / \mathrm{N} / \uparrow / \mathrm{N} / \mathrm{N}$ & $\begin{array}{c}\text { Pasialo, disialo } \\
\downarrow \text { trisialo, tetrasialo }\end{array}$ & NA \\
\hline 9/057/F/15 yr(s) [2] & $\begin{array}{c}\text { KCNA2 disease }(10 \mathrm{yr}(\mathrm{s})) \\
\text { by ES }\end{array}$ & $\begin{array}{c}8 \mathrm{mo}(\mathrm{s}) / \mathrm{GTCS}, \mathrm{GTS}, \\
\mathrm{MS}, \mathrm{AbS}\end{array}$ & GDD, ataxia & PB, LVT, VPA, OXC & LVT, VPA & NA/NA/NA/NA/NA/NA & $\uparrow$ trisialo & $\begin{array}{l}\text { Pat N } \\
\text { Mat N }\end{array}$ \\
\hline $10 / 090 / \mathrm{F} / 7 \mathrm{yr}(\mathrm{s})[2]$ & $\begin{array}{c}\text { GRIN1 disease (7 yr(s)) by } \\
\text { ES }\end{array}$ & Day 7/GTCS, AbS & GDD, spastic diplegia & $\begin{array}{c}\text { PB, CBZ, VPA, CZP, } \\
\text { LOR }\end{array}$ & VPA, CZP & $\uparrow / \mathrm{NA} / \mathrm{NA} / \mathrm{N} / \mathrm{NA} / \mathrm{NA}$ & $\uparrow$ trisialo & Mat $N$ \\
\hline
\end{tabular}


Table 1. Cont

\begin{tabular}{|c|c|c|c|c|c|c|c|c|}
\hline $\begin{array}{c}\text { Patient Number/ Study } \\
\text { ID/Sex/Current Age } \\
\text { (Reference) }\end{array}$ & $\begin{array}{l}\text { Diagnosis (Genetic or } \\
\text { None) (Age of Diagnosis) }\end{array}$ & $\begin{array}{c}\text { Seizure Age of } \\
\text { Onset/Seizure Types }\end{array}$ & $\begin{array}{l}\text { Other Clinical } \\
\text { Features }\end{array}$ & $\begin{array}{c}\text { Anti-Epileptic } \\
\text { Medications Used }\end{array}$ & $\begin{array}{l}\text { Anti-Epileptic } \\
\text { Medications at the } \\
\text { Time of TIEF Test }\end{array}$ & $\begin{array}{c}\text { Liver Functions } \\
\text { AST/ALT/INR/GGT/ } \\
\text { ALP/Direct Bil }\end{array}$ & TIEF Test & Parental TIEF \\
\hline 11/102/F/17 yr(s) [2] & $\begin{array}{c}\text { EP300 disease (11 yr(s)) } \\
\text { by ES }\end{array}$ & $8 \mathrm{yr}(\mathrm{s}) / \mathrm{AbS}$ & $\begin{array}{c}\text { GDD, ASD, } \\
\text { dysmorphic features } \\
\text { (triangular shaped face } \\
\text { with prominent } \\
\text { eyebrows, thin upper } \\
\text { lip, narrow high arched } \\
\text { palate, narrow } \\
\text { forehead, posteriorly } \\
\text { rotated ears, } \\
\text { retrognathia, } \\
\text { prominent frontal } \\
\text { incisors) }\end{array}$ & VPA & VPA & N/N/NA/N/NA/NA & $\begin{array}{l}\uparrow \text { trisialo } \\
\downarrow \text { tetrasialo }\end{array}$ & Mat $\mathrm{N}$ \\
\hline 12/193/F/11 yr(s) & $\begin{array}{l}\text { HIVEP2 disease (6 yr(s)) } \\
\text { by ES }\end{array}$ & $\begin{array}{c}20 \mathrm{mo}(\mathrm{s}) / \mathrm{GTCS}, \mathrm{MS}, \\
\mathrm{AbS}\end{array}$ & GDD, ADHD & LVT, VPA & VPA, LVT & $\mathrm{N} / \mathrm{N} / \mathrm{NA} / \mathrm{NA} / \downarrow / \mathrm{N}$ & Tetrasialo doublet & NA \\
\hline 13/197/M/13 yr(s) [2] & $\begin{array}{c}\text { KCNQ2 disease }(7 \mathrm{yr}(\mathrm{s})) \\
\text { by TNGSP for epilepsy } \\
\text { (70 genes) }\end{array}$ & Day 1/GTCS, GTS & $\begin{array}{l}\text { GDD, dysmorphic } \\
\text { features (thick } \\
\text { eyebrows, flat nasal } \\
\text { bridge, prominent } \\
\text { philtral groove, malar } \\
\text { hypoplasia) }\end{array}$ & $\begin{array}{l}\text { PHT, PB, TPM, LOR, } \\
\text { MID, CLB }\end{array}$ & PB, TPM, CLB & NA/NA/NA/NA/NA/NA & $\uparrow$ trisialo & NA \\
\hline 14/016/M/3 yr(s) & None (ES negative) & $5 \mathrm{mo}(\mathrm{s}) / \mathrm{IS}$ & GDD & MID, VGB, TPM & None & $\uparrow / \uparrow / \mathrm{N} / \mathrm{N} / \uparrow / \mathrm{N}$ & $\begin{array}{c}\uparrow \text { asialo, disialo } \\
\downarrow \text { tetrasialo }\end{array}$ & $\begin{array}{l}\text { Pat N } \\
\text { Mat N }\end{array}$ \\
\hline $15 / 021 / \mathrm{M} / 4 \mathrm{yr}(\mathrm{s})$ & $\begin{array}{l}\text { None (TNGSP for } \\
\text { epilepsy, } 127 \text { genes) }\end{array}$ & $\begin{array}{c}10 \mathrm{mo}(\mathrm{s}) / \mathrm{GTS}, \mathrm{IS}, \mathrm{MS}, \\
\text { AbS, AS }\end{array}$ & GDD & $\begin{array}{l}\text { VPA, LOR, GBP, CBD, } \\
\text { CZP, TPM, VGB, ACTH }\end{array}$ & VPA, LOR, GBP & $\mathrm{N} / \mathrm{N} / \mathrm{NA} / \uparrow / \mathrm{N} / \mathrm{NA}$ & $\uparrow$ trisialo & $\mathrm{N} / \mathrm{A}$ \\
\hline 16/050/F/13 yr(s) & $\begin{array}{l}\text { None (TNGSP for } \\
\text { epilepsy, } 87 \text { genes) }\end{array}$ & $2 \mathrm{yr}(\mathrm{s}) / \mathrm{GTCS}, \mathrm{GTS}, \mathrm{MS}$ & GDD, ADHD & LVT, VPA, LOR & VPA & NA/NA/NA/NA/NA/NA & Tetrasialo doublet & N/A \\
\hline $17 / 059 / \mathrm{M} / 7 \mathrm{yr}(\mathrm{s})$ & None (ES negative) & $2 \mathrm{yr}(\mathrm{s}) / \mathrm{GTCS}, \mathrm{MS}, \mathrm{AS}$ & GDD & $\begin{array}{l}\text { LOR, LVT, CBZ, VPA, } \\
\text { CLB, TPM, FOS }\end{array}$ & CLB, TPM, VPA, LOR & $\mathrm{N} / \mathrm{N} / \uparrow / \mathrm{N} / \downarrow / \mathrm{NA}$ & $\uparrow$ trisialo & $\begin{array}{l}\text { Pat N } \\
\text { Mat N }\end{array}$ \\
\hline 18/064/M/16 yr(s) & None (ES negative) & $\begin{array}{c}18 \mathrm{mo}(\mathrm{s}) / \mathrm{GTCS}, \mathrm{MS} \\
\mathrm{AbS}\end{array}$ & ASD & $\begin{array}{l}\text { VPA, CBZ, ESM, LMT, } \\
\text { LVT, TPM, CLB, RUF }\end{array}$ & VPA & NA/NA/NA/NA/NA/NA & $\uparrow$ trisialo & $\begin{array}{l}\text { Pat N } \\
\text { Mat N }\end{array}$ \\
\hline 19/066/M/16 yr(s) & None (microarray) & $2.5 \mathrm{yr}(\mathrm{s}) / \mathrm{MS}, \mathrm{AbS}$ & $\begin{array}{c}\text { Tremor, ADHD, ASD, } \\
\text { temper dysregulation } \\
\text { disorder, aggressive } \\
\text { behaviour }\end{array}$ & $\begin{array}{l}\text { VPA, CBZ, CLB, ESM, } \\
\text { DZP }\end{array}$ & VPA & NA/NA/NA/NA/NA/NA & $\begin{array}{c}\uparrow \text { trisialo } \\
\downarrow \text { tetrasialo }\end{array}$ & $\mathrm{N} / \mathrm{A}$ \\
\hline 20/097/F/15 yr(s) & $\begin{array}{l}\text { None (TNGSP for } \\
\text { epilepsy, } 87 \text { genes) }\end{array}$ & $6 \mathrm{yr}(\mathrm{s}) / \mathrm{GTCS}, \mathrm{MS}, \mathrm{CPS}$ & ADHD & VPA, LMT, TPM, ESM & VPA, LMT & NA/NA/N/NA/NA/NA & $\uparrow$ trisialo & $\begin{array}{l}\text { Pat: } \downarrow \text { asialo, } \\
\text { disialo, } \\
\downarrow \text { tetrasialo } \\
\text { Mat N }\end{array}$ \\
\hline
\end{tabular}


Table 1. Cont.

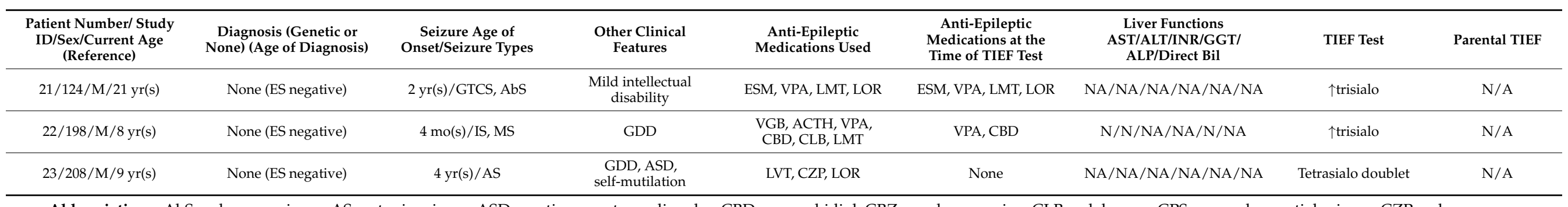

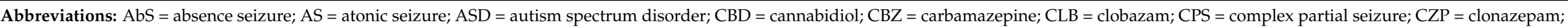

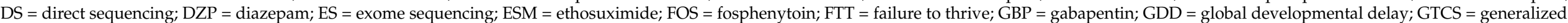

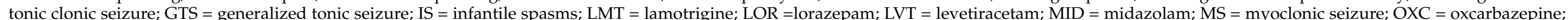

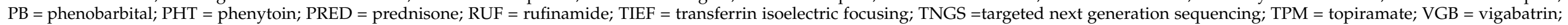
$\mathrm{VPA}=$ valproic acid; $\mathrm{N}=$ normal; $\mathrm{NA}=$ not available; $\uparrow=$ increased; $\downarrow$ = decreased; Pat = paternal; Mat = maternal. 


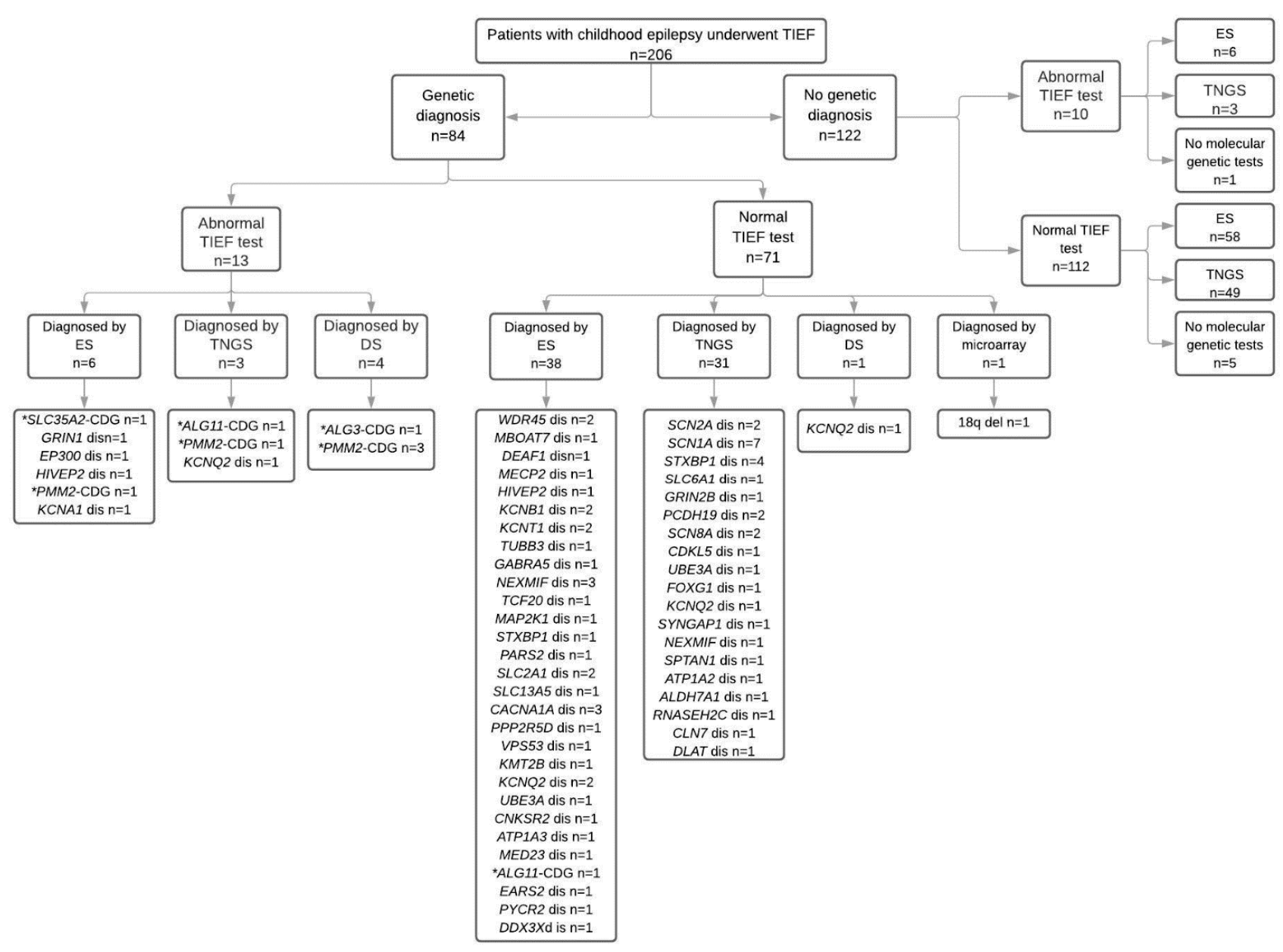

Figure 1. Number of patients with normal and abnormal TIEF tests, their genetic diagnosis and type of molecular genetic investigation are depicted in Figure $1 .{ }^{*}$ Statistically significant.

The TIEF test was abnormal in 11\% (23 out of 206) of the patients. In $35 \%$ (8 out of 23 ) of those patients, CDG genetic diagnoses were confirmed by different genetic investigations. We used the current nomenclature for CDG, gene-CDG for all subgroups of CDG throughout the manuscript. There were eight patients with different types of CDG including PMM2CDG $(n=5), \operatorname{ALG3-CDG}(n=1), \operatorname{ALG11-CDG}(n=1)$, and SLC35A2-CDG $(n=1)$. In $22 \%$ (5 out of 23 ) of the patients with an abnormal TIEF test, non-CDG genetic diagnoses were confirmed, including EP300 disease $(n=1)$, GRIN1 disease $(n=1)$, HIVEP2 disease $(n=1), K C N A 2$ disease $(n=1)$, and $K C N Q 2$ disease $(n=1)$. All variants are summarized in Supplemental Tables S1 and S3. In the remaining 10 patients, only six of them had ES which was normal. Three patients had TNGSP for epilepsy which included only one CDG gene called ALG13. The false positive rate of the TIEF test was 5.3\% (11 out of 206) in the study cohort. The false negative TIEF test rate was $0.5 \%$ ( 1 out of 206 ).

In the study cohort, CDG genetic diagnoses were confirmed in $4 \%$ of the patients (9 out of 206) by (1) an abnormal TIEF test and direct sequencing of PMM2 or ALG3 $(n=4)$; (2) an abnormal TIEF test and targeted next generation sequencing panel for CDG $(n=2)$; (3) an abnormal TIEF test and ES $(n=2)$; (4) a normal TIEF test and ES $(n=1)$. All variants are summarized in Supplemental Tables S1 and S3.

One hundred and eight (52.4\%) patients underwent ES, and its diagnostic yield was $40.7 \%$ (44 out of 108) in the study cohort. One hundred and sixty-six patients underwent 
TNGSP, and its diagnostic yield was 20.5\% (34 out of 166) in the study cohort. One hundred and ninety-eight patients underwent microarray, and its diagnostic yield was $0.5 \%$ (1 out of 198 patients).

There were 51 different genetic diagnoses in 84 patients (48 previously published and 36 new patients) (Supplemental Tables S1 and S2). All genetic diagnoses and the number of patients for each genetic disease are depicted in Figure 2. Interestingly more than half (44 out of 84 ) of the patients were diagnosed by ES. The remaining patients were diagnosed by various types of molecular genetic investigations including TNGSP for epilepsy $(n=29)$, for Aicardi-Goutières Syndrome $(n=1)$, for Cornelia de Lange syndrome $(n=1)$, for neuronal ceroid lipofuscinosis $(n=1)$, for pyruvate dehydrogenase complex $(n=1)$, for CDG $(n=2)$, and targeted Sanger sequencing of candidate genes $(n=5)$. One hundred and twenty-two $(59.2 \%)$ patients had no genetic diagnosis and underwent ES $(n=64)$, and TNGSP $(n=52)$.

One hundred and two patients had at least one liver function test performed, including liver enzymes (AST and/or ALT $n=99$ ), liver function tests for cholestasis (ALP and/or GGT and/or bilirubin $n=80)$, and/or INR $(n=24)$. Twenty-nine patients had elevated AST and/or ALT including with a normal $(n=24)$ TIEF test and an abnormal $(n=5)$ TIEF test. Eight patients had elevated ALP and/or GGT and/or bilirubin including with a normal $(n=3)$ TIEF test and an abnormal $(n=5)$ TIEF test. Eight patients had elevated INR including with a normal $(n=3)$ TIEF test and an abnormal $(n=2)$ TIEF test. There was no association between abnormal liver functions and abnormal TIEF tests ( $p$ value of 0.9904). There was no association between anti-epileptic medications and abnormal TIEF test ( $p$ value of 0.544 ). There was no association between abnormal liver functions and anti-epileptic medications ( $p$ value of 0.9959 ).

There were 93 variants in 50 different genes in 83 patients (one patient was diagnosed with 18qdel syndrome by microarray). Forty-eight patients were reported previously [2,4,7]. The ACMG variant classification of 43 patients were reported previously too [2,7]. In silico analysis and ACMG variant classification of the remaining 35 patients (additionally five previously published patients by Al Teneiji et al. [4]) are listed in Supplemental Table S3. There were 46 different variants from 40 patients (excluding patients with microarray abnormality), including 24 novel and 22 known variants. According to ACMG variant classification, 22 variants were pathogenic, 17 variants were likely pathogenic, and 7 variants were variants of uncertain significance. Segregation was confirmed in both parents in 30 patients and in one parent in four patients and in none of the parents in six patients.

We grouped patients into two groups: (1) abnormal TIEF test; (2) normal TIEF test. We compared both groups for their demographics, seizure types, anti-epileptic medications, and liver functions to see if there was any difference between both groups (Table 2). Interestingly, there were statistically significant differences between groups for liver functions including ALT, AST, and GGT, which was elevated in the group with abnormal TIEF tests. Additionally, there was a statistically significant difference between groups for valproic acid treatment with valproic acid being more commonly used in the group with abnormal TIEF tests.

We grouped patients into two groups, including: (1) with genetic diagnosis; (2) with no genetic diagnosis, and compared both groups for their demographics, seizure types, anti-epileptic medications, liver functions, and TIEF test results to see if there was any difference between groups (Table 3). There was a statistically significant difference for atonic seizures in the group with no genetic diagnosis. There was also a statistically significant difference for elevated asialotransferrin and disialotransferrin in the group with genetic diagnosis, which is likely associated with different types of CDG diagnosis in the group with genetic diagnosis. 


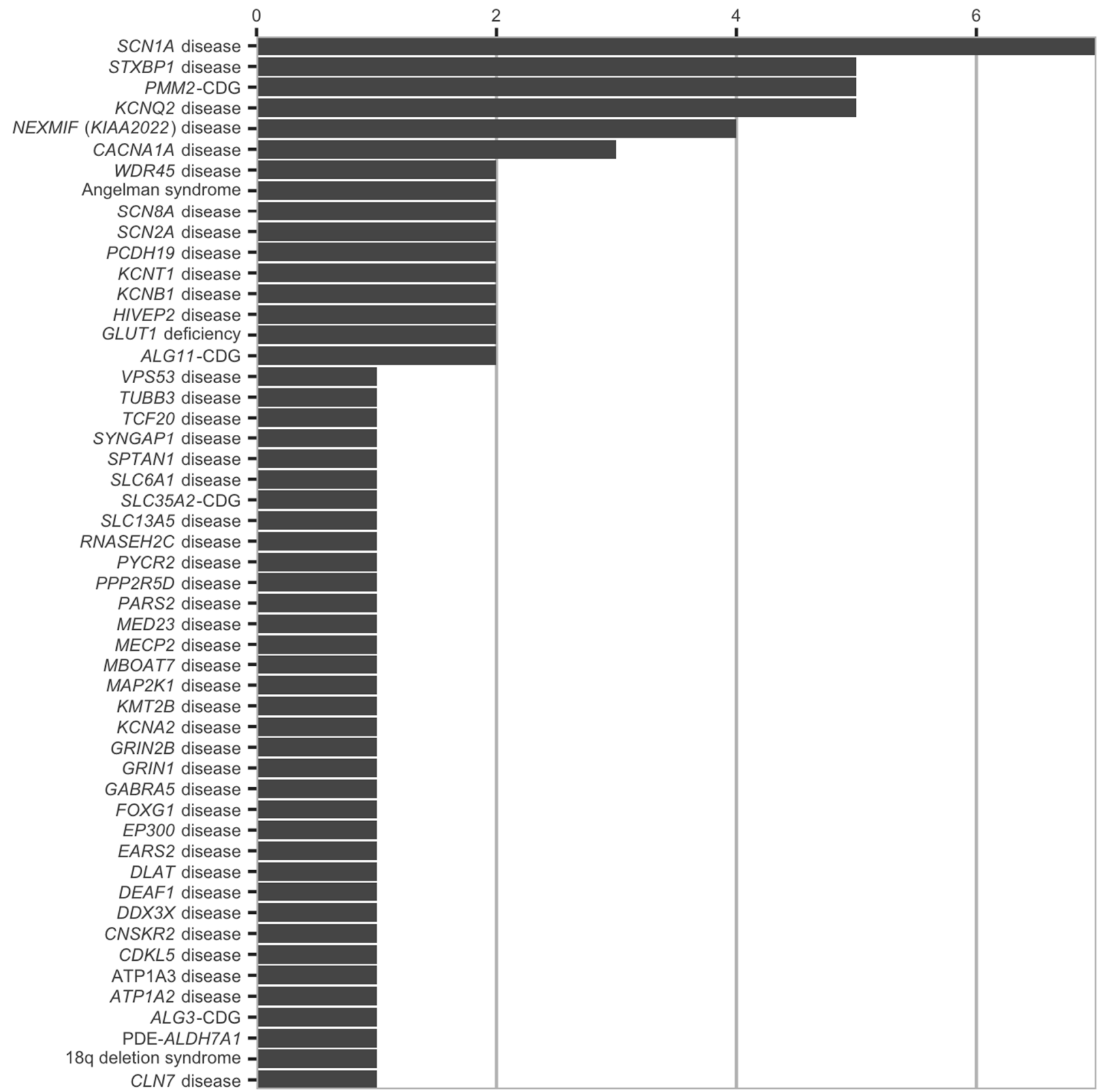

Figure 2. All genetic diagnoses and the number of patients for each genetic disease are depicted in Figure 2. 
Table 2. Comparison of patients with abnormal or normal transferrin isoelectric focusing for their demographics, seizure types, anti-epileptic medications, and liver functions.

\begin{tabular}{|c|c|c|c|c|c|}
\hline & \multicolumn{2}{|c|}{$\begin{array}{l}\text { With Abnormal TIEF } \\
\qquad(n=23)\end{array}$} & \multicolumn{2}{|c|}{$\begin{array}{l}\text { With Normal TIEF } \\
\quad(n=183)\end{array}$} & \multirow[t]{6}{*}{$\begin{array}{c}p \text {-Value (Fisher } \\
\text { Exact Test) }\end{array}$} \\
\hline $\begin{array}{c}\text { Median Age at } \\
\text { Diagnosis (Months) }\end{array}$ & \multicolumn{2}{|c|}{60} & \multicolumn{2}{|c|}{60} & \\
\hline $\begin{array}{l}\text { Median Age at Onset } \\
\text { (Months) }\end{array}$ & \multicolumn{2}{|c|}{18} & \multicolumn{2}{|c|}{18} & \\
\hline & $n$ & $\%$ & $n$ & $\%$ & \\
\hline Sex (=Male) & 12 & 52.17 & 90 & 49.18 & \\
\hline \multicolumn{5}{|c|}{ Liver function tests } & \\
\hline AST & 6 & 26.09 & 19 & 10.38 & $0.04164 *$ \\
\hline ALT & 5 & 21.74 & 11 & 6.01 & $0.02111 *$ \\
\hline GGT & 5 & 21.74 & 2 & 1.09 & $0.0002055^{*}$ \\
\hline ALP & 0 & 0 & 1 & 0.55 & 1 \\
\hline Bilirubin & 0 & 0 & 2 & 1.09 & 1 \\
\hline INR & 2 & 8.70 & 3 & 1.64 & 0.09693 \\
\hline \multicolumn{6}{|c|}{ Anti-epileptic medications } \\
\hline Topiramate & 4 & 17.39 & 24 & 13.11 & 0.526830097 \\
\hline Phenobarbitone & 3 & 13.04 & 39 & 21.31 & 0.425122663 \\
\hline Clonazepam & 1 & 4.35 & 8 & 4.37 & 1 \\
\hline Carbamazepine & 0 & 0 & 11 & 6.01 & 0.615961230 \\
\hline Clobazam & 3 & 13.04 & 54 & 29.51 & 0.136747826 \\
\hline Lorazepam & 4 & 17.39 & 26 & 14.21 & 0.752853085 \\
\hline Valproic acid & 12 & 52.17 & 38 & 20.77 & $0.003085312 *$ \\
\hline Oxcarbazepine & 0 & 0 & 8 & 4.37 & 0.601562786 \\
\hline CBD oil & 1 & 4.35 & 7 & 3.83 & 1 \\
\hline Gabapentine & 1 & 4.35 & 0 & 0 & 0.111650485 \\
\hline Diazepam & 0 & 0 & 2 & 1.09 & 1 \\
\hline Vigabatrin & 1 & 4.35 & 4 & 2.19 & 0.450170633 \\
\hline Ethosuximide & 1 & 4.35 & 9 & 4.92 & 1 \\
\hline $\mathrm{ACTH}$ & 0 & 0 & 3 & 1.64 & 1 \\
\hline Acetazolamide & 0 & 0 & 1 & 0.55 & 1 \\
\hline Rufinamide & 0 & 0 & 2 & 1.09 & 1 \\
\hline Perampenil & 0 & 0 & 1 & 0.55 & 1 \\
\hline Midazolam & 0 & 0 & 3 & 1.64 & 1 \\
\hline Phenytoin & 0 & 0 & 4 & 2.19 & 1 \\
\hline Stiripentol & 0 & 0 & 1 & 0.55 & 1 \\
\hline Lacosamide & 0 & 0 & 1 & 0.55 & 1 \\
\hline \multicolumn{6}{|c|}{ Types of seizures } \\
\hline Generalized seizures & 17 & 73.91 & 149 & 81.42 & 0.4047 \\
\hline Partial seizures & 3 & 13.04 & 57 & 31.15 & 0.08905 \\
\hline Infantile spasms & 5 & 21.74 & 34 & 18.58 & 0.7776 \\
\hline Absence seizures & 8 & 34.78 & 67 & 36.61 & 1 \\
\hline Atonic seizures & 3 & 13.04 & 50 & 27.32 & 0.205 \\
\hline Myoclonic seizures & 10 & 43.48 & 63 & 34.43 & 0.4883 \\
\hline
\end{tabular}

* Statistically significant. 
Table 3. Comparison of patients with and without a genetic diagnosis for their demographics, seizure types, anti-epileptic medications, transferrin isoelectric focusing and liver functions.

\begin{tabular}{|c|c|c|c|c|c|}
\hline Clinical Features \& Results & \multicolumn{2}{|c|}{$\begin{array}{l}\text { With Genetic Diagnosis } \\
\qquad(n=84)\end{array}$} & \multicolumn{2}{|c|}{$\begin{array}{l}\text { Without Genetic Diagnosis } \\
\qquad(n=122)\end{array}$} & \multirow[t]{6}{*}{$\begin{array}{c}p \text {-Value (Fisher Exact } \\
\text { Test) }\end{array}$} \\
\hline Median Age at Diagnosis (Months) & \multicolumn{2}{|c|}{60} & \multicolumn{2}{|c|}{60} & \\
\hline \multirow[t]{2}{*}{ Median Age at Onset (Months) } & \multicolumn{2}{|c|}{18} & \multicolumn{2}{|c|}{18} & \\
\hline & $\mathrm{N}$ & $\%$ & $\mathrm{~N}$ & $\%$ & \\
\hline Sex (=Male) & 33 & 39.29 & 69 & 56.56 & \\
\hline \multicolumn{5}{|c|}{ Liver function tests } & \\
\hline AST & 15 & 17.86 & 10 & 8.20 & 0.04985895 \\
\hline ALT & 8 & 9.52 & 8 & 6.56 & 0.44088861 \\
\hline GGT & 5 & 5.95 & 2 & 1.64 & 0.12384944 \\
\hline ALP & 0 & 0 & 1 & 0.82 & 1 \\
\hline Bilirubin & 1 & 1.19 & 1 & 0.82 & 1 \\
\hline INR & 2 & 2.38 & 3 & 2.46 & 1 \\
\hline \multicolumn{6}{|c|}{ Anti-epileptic medications } \\
\hline Levetiracetam & 32 & 38.10 & 31 & 25.41 & 0.06483421 \\
\hline Topiramate & 9 & 10.71 & 19 & 15.57 & 0.40901063 \\
\hline Phenobarbitone & 22 & 26.19 & 20 & 16.39 & 0.11280733 \\
\hline Clonazepam & 4 & 4.76 & 5 & 4.10 & 1 \\
\hline Carbamazepine & 5 & 5.95 & 6 & 4.92 & 1 \\
\hline Clobazam & 20 & 23.81 & 37 & 30.33 & 0.34373283 \\
\hline Lorazepam & 14 & 16.68 & 16 & 13.11 & 0.54802867 \\
\hline Valproate & 20 & 23.81 & 30 & 24.59 & 1 \\
\hline Oxcarbazepine & 3 & 3.57 & 5 & 4.10 & 1 \\
\hline CBD oil & 3 & 3.57 & 5 & 4.10 & 1 \\
\hline Gabapentin & 0 & 0 & 1 & 0.82 & 1 \\
\hline Diazepam & 1 & 1.19 & 1 & 0.82 & 1 \\
\hline Vigabatrin & 1 & 1.19 & 4 & 3.28 & 0.65038894 \\
\hline Ethosuximide & 1 & 1.19 & 9 & 7.38 & 0.05042569 \\
\hline АCTH & 0 & 0 & 3 & 2.46 & 0.27198674 \\
\hline Acetazolamide & 1 & 1.19 & 0 & 0 & 0.40776699 \\
\hline Rufinamide & 0 & 0 & 2 & 1.64 & 0.51465783 \\
\hline Perampenil & 0 & 0 & 1 & 0.82 & 1 \\
\hline Midazolam & 1 & 1.19 & 2 & 1.64 & 1 \\
\hline Phenytoin & 2 & 2.38 & 2 & 1.64 & 1 \\
\hline Stiripentol & 1 & 1.19 & 0 & 0 & 0.40776699 \\
\hline Lacosamide & 1 & 1.19 & 0 & 0 & 0.40776699 \\
\hline \multicolumn{6}{|c|}{ Type of Seizures } \\
\hline Generalized Seizures & 69 & 82.14 & 97 & 79.51 & 0.72135379 \\
\hline Partial Seizures & 26 & 30.95 & 34 & 27.87 & 0.64291817 \\
\hline Infantile Spasms & 11 & 13.10 & 28 & 22.95 & 0.10274649 \\
\hline Absence Seizures & 24 & 28.57 & 51 & 41.80 & 0.05687798 \\
\hline Atonic Seizures & 15 & 17.86 & 38 & 31.15 & $0.03568696^{*}$ \\
\hline Myoclonic Seizures & 26 & 30.95 & 47 & 38.52 & 0.30083751 \\
\hline \multicolumn{6}{|c|}{ TIEF test } \\
\hline Asialotransferrin & 5 & 5.95 & 1 & 0.82 & $0.042312860 *$ \\
\hline Monosialotransferrin & 1 & 1.19 & 0 & 0 & 0.407766990 \\
\hline Disialotransferrin & 7 & 8.33 & 1 & 0.82 & $0.008505866^{*}$ \\
\hline Trisialotransferrin & 5 & 5.95 & 7 & 5.74 & 1 \\
\hline Tetrasialotransferrin & 7 & 8.33 & 4 & 3.28 & 0.126938204 \\
\hline Pentasialotransferrin & 0 & 0 & 1 & 0.82 & 1 \\
\hline
\end{tabular}




\section{Discussion}

The prevalence of CDG was $4.4 \%$ in childhood epilepsy in our study. We report nine patients with four different CDG. We found a significant correlation between abnormal liver enzymes and abnormal TIEF test. We also found a significant correlation between valproic acid use and an abnormal TIEF test. Interestingly, the false positive rate of the TIEF test was $5.3 \%$ with a small percentage of false negative TIEF test rate $(0.5 \%)$ in our study cohort. To the best of our knowledge, our study is the first study reporting the prevalence of CDG in childhood epilepsy and an abnormal TIEF test possibly related to anti-epileptic medications.

Hepatotoxicity associated with anti-epileptic medications is well known and has been summarized in review articles previously. Phenytoin, carbamazepine, valproic acid, and lamotrigine are commonly reported for their hepatoxic side-effects [8,9]. Valproic acid use is also associated with hyperammonemia and encephalopathy, toxic hepatitis, and Reye-like syndrome [8,9]. Carbamazepine and phenytoin-associated hepatotoxicity is characterized by asymptomatic transient elevations in liver enzymes occurring in up to $75 \%$ of patients [9]. Severe hepatocellular injury leading to liver failure has been reported in phenytoin, carbamazepine, and valproic acid use as the most severe spectrum of hepatotoxicity $[9,10]$. Most hepatotoxic reactions are idiosyncratic or immune hypersensitivity reactions [8,9]. Reactive arene oxide metabolites of phenytoin, carbamazepine, valproic acid, and lamotrigine are thought to be important in hepatotoxicity where they may cause oxidative injury and secondary immune response $[8,9]$. Valproic acid specifically is thought to cause hepatotoxicity through the inhibition of mitochondrial $\beta$-oxidation by reactive metabolites [8,9]. Frequency of valproic acid hepatoxicity is up to 1 in 800 in children under the age of two years [11]. In our study, one-quarter of the patients had abnormal liver functions. There was no significant association between abnormal liver functions and anti-epileptic medications ( $p$ value 0.9959$)$ and between anti-epileptic medications and abnormal TIEF test ( $p$ value 0.544 ).

Abnormal TIEF test have been associated with liver disease or inherited metabolic disorders affecting liver functions, such as hereditary fructose intolerance and galactosemia $[12,13]$. In a recent study, 1546 individuals underwent TIEF test [14]. An abnormal TIEF test was identified in 3\% (51 out of 1546) of those individuals. There were follow-up investigations in 14 of those patients. Only four patients received a confirmed diagnosis of CDG: PMM2-CDG $(n=2)$, MPDU1-CDG $(n=1)$, and SLC35A2-CDG $(n=1)$, whereas in 10 patients, an abnormal TIEF test was secondary to other inherited metabolic disorders: galactosemia $(n=4)$, hereditary fructose intolerance $(n=2)$, and peroxisomal disease $(n=2)$. Individuals with liver disease had a 4.6 times higher likelihood of abnormal TIEF test results [14]. In another study, 554 children with a suspected CDG underwent TIEF test and nine of them had an abnormal TIEF test. Four patients had PMM2-CDG, and two patients had ALG2-CDG [15]. In our study, 4.4\% (9 out of 206) of the patients had genetically confirmed CDG and most of those patients had an abnormal TIEF test to suggest CDG. We identified other inherited metabolic disorders including pyridoxine-dependent epilepsy (PDE) due to biallelic variants in ALDH7A1 [4], PYCR2 disease [16], EARS2 disease [17], PARS2 disease [17], CLN7 disease, and glucose transporter 1 deficiency syndrome with or without liver dysfunction in our study cohort. We did not identify any patients with galactosemia or hereditary fructose intolerance in our study cohort. We think that the TIEF test might be included into the diagnostic investigations as screening test of CDG.

In a recent study, glycosylation was studied in 981 patients with adult liver disease to investigate if there was any difference between primary and secondary glycosylation defects in liver disease. An abnormal TIEF test was identified in $26 \%$ of the patients, including increased a percentage of trisialotransferrin (70\%), monosialotransferrin (17\%), disialotransferrin (4\%) and mixed transferrin isoforms (11\%) [18]. None of the patients had a CDG diagnosis [18]. There is no information for neurological phenotypes of those patients and trisiaolotransferrin is not one of the typical CDG type I or II patterns. The study had no information regarding the underlying causes of liver disease [18]. In another 
study, 19 pediatric liver disease patients underwent a TIEF test [19]; 17 of them had elevated asialotransferrin and monosialotransferrin, and 13 of them had elevated disialotransferrin. Eight patients had follow-up liver function tests and TIEF test were normalized in all eight patients during follow-up. Two patients had inherited metabolic disorders: citrin deficiency $(n=1)$ and deoxyguanosine kinase deficiency $(n=1)$ [19]. Our patient population is unique, as we did not select patients with liver disease, but included all patients with epilepsy who had TIEF test for diagnostic investigations.

Transferrin is synthesized by the endoplasmic reticulum and golgi apparatus in the liver. Hepatocyte injury results in endoplasmic reticulum damage and impaired excretory function of glycosylated proteins [19]. Changes in the TIEF test profile in liver disease represent alterations occurring during biosynthesis of transferrin by the endoplasmic reticulum (ER) and golgi apparatus [20]. Several changes have been reported in individuals with liver disease: (1) chronic alcohol abuse is known to alter the glycosylation pattern with an increase in asialotransferrin and disialotransferrin [21]; (2) polymorphisms in transferrin B2 and C2 may result in abnormal TIEF test characterized by elevated pentasialotransferrin and trisialotransferrin [21]; (3) fucosyltransferase activity and fucosylation are increased in alcoholic liver disease, cholestatic liver disease, and hepatocellular carcinoma [20]; (4) sialyltransferase, galactosyltransferase, and mannosyltransferase activity changes have been reported in alcoholic liver disease $[18,19]$. It has been shown that valproic acid impaired glycosylation and the secretion of acid phosphatase in fission yeast model. The authors speculated that this could contribute to adverse effects of valproic acid in humans [22]. Taken together, liver injury affects glycosylation. There might be a causative link for the abnormal glycosylation associated with anti-epileptic treatment and their effects on the cellular and synthetic liver functions in childhood epilepsy.

When proteins and lipids are combined with sugars, advanced glycation end-products (AGE) are generated which are implicated to contribute to disease progression. Receptor for AGE (RAGE) is found in different cells. When RAGE binds to AGE, nuclear factor kappa B (NFkB) is activated and releases inflammatory mediators, which contributes to inflammation, intracellular damage and apoptosis [23-25]. In brain biopsy samples of individuals with temporal lobe epilepsy, RAGE was upregulated in astrocytes, neurons, and microvessels and was reported to contribute to seizures [23]. In seizing mice, RAGE was upregulated, while RAGE knockout mice had reduced seizure activity [23]. The presence of G28S polymorphism in RAGE was significantly more common in patients with drug-resistant epilepsy in a study [25]. In individuals with epilepsy, increased blood sugar levels have been reported, which may be associated with AGE and poor seizure control in childhood epilepsy. While a mechanism for abnormal glycosylation associated with epilepsy and antiepileptic treatment is unknown, we propose a possible link with RAGE.

Post-translational modification is a protein modification process to improve structure and function of proteins after protein synthesis. Interestingly EP300, GRIN1, KCNA2, and KCNQ2 proteins undergo post-translational modification either by amino acid modifications and N-linked glycosylation (KCNA2 and GRIN1 proteins) or ubiquitylation and O-glycosylation (KCNQ2 and EP300 proteins). It is likely that pathogenic or likely pathogenic variants in these genes may affect the post-translational modification of these proteins via $\mathrm{N}$ - or O-glycosylation and cause abnormal TIEF test. Further research is necessary to understand better the implications of the variants on the post-translational modification of proteins.

Limitations of our study include: (1) a retrospective cohort study that reports patients with epilepsy seen in a single epilepsy genetics clinic for diagnostic investigations; (2) exome sequencing was not applied to all patients; (3) the study was initiated in the metabolic unit; this has likely contributed to the higher number of patients with CDG; (4) there might have been other CDG patients at our institution who were diagnosed during our study period that were not included into this study, as they were not known to the authors; (5) we have no patient database to perform an institution-wide retrospective study for all patients with epilepsy who underwent transferrin isoelectric focusing; (6) we do not know the 
diagnostic yield of the TIEF test at our institution. Despite these limitations, we think that our study results provide a diagnostic yield of TIEF test and a high prevalence of CDG in childhood epilepsy.

In summary, interestingly, the TIEF test guided CDG diagnosis in $2.9 \%$ (6 out of 206) of the patients and prevented the use of untargeted molecular genetic investigations such as ES. Due to the high prevalence of CDG in childhood epilepsy, the TIEF test might be included in the diagnostic investigations. Application of the TIEF test in childhood epilepsy may allow physicians to reach a diagnosis in a shorter period of time and be cost effective.

Supplementary Materials: The following are available online at https: / www.mdpi.com/article/ 10.3390 /genes12081227/s1, Table S1: Clinical features, neuroimaging, and genetic test results of all patients with genetically confirmed childhood epilepsy identified in this study are summarized. Table S2: Please refer to our previously published studies for the results of the 48 patients with genetic diagnoses, who were included into the current study. Table S3: In silico analysis of variants in genes identified in patients with childhood epilepsy are listed in Table S3.

Author Contributions: Conception and design, acquisition of data, or analysis and interpretation of data: G.S., S.B., D.C., A.T., T.A., S.M.-A. Drafting the article or revising it critically for important intellectual content: G.S., S.B., S.M.-A. Final approval of the version to be published: all authors (G.S., S.B., D.C., A.T., T.A., S.M.-A.). All authors have read and agreed to the published version of the manuscript.

Funding: No financial assistance was received in support of the study.

Institutional Review Board Statement: The study was conducted according to the guidelines of the Declaration of Helsinki and approved by the Institutional Research Ethics Board of The Hospital for Sick Children (protocol code/approval number \#1000070163 and approval date on 24-May-2020).

Informed Consent Statement: No patient consent was required.

Data Availability Statement: Not applicable.

Acknowledgments: The authors thank the referring physicians and genetic counsellors for providing genetic counseling to the families. We would like to thank the patients' families for their dedication to the diagnostic investigations.

Conflicts of Interest: The authors declare no competing interest.

\section{References}

1. Mercimek-Mahmutoglu, S.; Patel, J.; Cordeiro, D.; Hewson, S.; Callen, D.; Donner, E.J.; Hahn, C.; Kannu, P.; Kobayashi, J.; Minassian, B.A.; et al. Diagnostic yield of genetic testing in epileptic encephalopathy in childhood. Epilepsia 2015, 56, 707-716. [CrossRef]

2. Costain, G.; Cordeiro, D.; Matviychuk, D.; Mercimek-Andrews, S. Clinical Application of Targeted Next-Generation Sequencing Panels and Whole Exome Sequencing in Childhood Epilepsy. Neuroscience 2019, 418, 291-310. [CrossRef]

3. Jaeken, J.; van Eijk, H.; van der Heul, C.; Corbeel, L.; Eeckels, R.; Eggermont, E. Sialic acid-deficient serum and cerebrospinal fluid transferrin in a newly recognized genetic syndrome. Clin. Chim. Acta 1984, 144, 245-247. [CrossRef]

4. Al Teneiji, A.; Bruun, T.U.; Sidky, S.; Cordeiro, D.; Cohn, R.D.; Mendoza-Londono, R.; Moharir, M.; Raiman, J.; Siriwardena, K.; Kyriakopoulou, L.; et al. Phenotypic and genotypic spectrum of congenital disorders of glycosylation type I and type II. Mol. Genet. Metab. 2017, 120, 235-242. [CrossRef]

5. Richards, S.; Aziz, N.; Bale, S.; Bick, D.; Das, S.; Gastier-Foster, J.; Grody, W.W.; Hegde, M.; Lyon, E.; Spector, E.; et al. Standards and guidelines for the interpretation of sequence variants: A joint consensus recommendation of the American College of Medical Genetics and Genomics and the Association for Molecular Pathology. Genet. Med. 2015, 17, 405-423. [CrossRef]

6. Lek, M.; Karczewski, K.J.; Minikel, E.V.; Samocha, K.E.; Banks, E.; Fennell, T.; O’Donnell-Luria, A.H.; Ware, J.S.; Hill, A.J.; Cummings, B.B.; et al. Analysis of protein-coding genetic variation in 60,706 humans. Nature 2016, 536, 285-291. [CrossRef]

7. Jilani, A.; Matviychuk, D.; Blaser, S.; Dyack, S.; Mathieu, J.; Prasad, A.N.; Prasad, C.; Kyriakopoulou, L.; Mercimek-Andrews, S. High diagnostic yield of direct Sanger sequencing in the diagnosis of neuronal ceroid lipofuscinoses. JIMD Rep. 2019, 50, 20-30. [CrossRef] [PubMed]

8. Björnsson, E. Hepatotoxicity associated with antiepileptic drugs. Acta Neurol. Scand. 2008, 118, 281-290. [CrossRef] [PubMed]

9. Vidaurre, J.; Gedela, S.; Yarosz, S. Antiepileptic Drugs and Liver Disease. Pediatr. Neurol. 2017, 77, 23-36. [CrossRef] 
10. Koenig, S.A.; Buesing, D.; Longin, E.; Oehring, R.; Häussermann, P.; Kluger, G.; Lindmayer, F.; Hanusch, R.; Degen, I.; Kuhn, H.; et al. Valproic acid-induced hepatopathy: Nine new fatalities in Germany from 1994 to 2003. Epilepsia 2006, 47, 2027-2031. [CrossRef] [PubMed]

11. Quintana, E.; Sturiale, L.; Montero, R.; Andrade, F.; Fernandez, C.; Couce, M.L.; Barone, R.; Aldamiz-Echevarria, L.; Ribes, A.; Artuch, R.; et al. Secondary disorders of glycosylation in inborn errors of fructose metabolism. J. Inherit. Metab. Dis. 2009, 32 (Suppl. 1), 273-278. [CrossRef] [PubMed]

12. Sturiale, L.; Barone, R.; Fiumara, A.; Perez, M.; Zaffanello, M.; Sorge, G.; Pavone, L.; Tortorelli, S.; O’Brien, J.F.; Jaeken, J.; et al. Hypoglycosylation with increased fucosylation and branching of serum transferrin N-glycans in untreated galactosemia. Glycobiology 2005, 15, 1268-1276. [CrossRef]

13. Magalhães, A.P.P.S.D.; Burin, M.G.; Souza, C.F.M.D.; de Bitencourt, F.H.; Sebastião, F.M.; Silva, T.O.; Schwartz, I.V.D. Transferrin isoelectric focusing for the investigation of congenital disorders of glycosylation: Analysis of a ten-year experience in a Brazilian center. J. Pediatr. 2020, 96, 710-716. [CrossRef] [PubMed]

14. Asteggiano, C.G.; Papazoglu, G.M.; Millón, M.B.B.; Peralta, M.F.; Azar, N.B.; Spécola, N.S.; Guelbert, N.; Suldrup, N.S.; Pereyra, M.; De Kremer, R.D. Ten years of screening for congenital disorders of glycosylation in Argentina: Case studies and pitfalls. Pediatr. Res. 2018, 84, 837-841. [CrossRef] [PubMed]

15. Afroze, B.; Mercimek-Andrews, S. Pyrroline-5-Carboxylate Reductase 2 Deficiency: A New Case and Review of the Litera-ture. Can. J. Neurol. Sci. 2020, 47, 280-282. [CrossRef]

16. Al Balushi, A.; Matviychuk, D.; Jobling, R.; Salomons, G.S.; Blaser, S.; Mercimek-Andrews, S. Phenotypes and genotypes of mitochondrial aminoacyl-tRNA synthetase deficiencies from a single neu-rometabolic clinic. JIMD Rep. 2020, 51, 3-10. [CrossRef]

17. Jansen, J.C.; Van Hoek, B.; Metselaar, H.J.; Berg, A.P.V.D.; Zijlstra, F.; Huijben, K.; Van Scherpenzeel, M.; Drenth, J.P.H.; Lefeber, D.J. Screening for abnormal glycosylation in a cohort of adult liver disease patients. J. Inherit. Metab. Dis. 2020, 43, 1310-1320. [CrossRef]

18. Bogdańska, A.; Lipiński, P.; Szymańska-Rożek, P.; Jankowska, I.; Socha, P.; Tylki-Szymańska, A. Pediatric Liver Disease Patients and Secondary Glycosylation Abnormalities. Front. Pediatr. 2021, 8, 613224. [CrossRef]

19. Blomme, B.; Van Steenkiste, C.; Callewaert, N.; Van Vlierberghe, H. Alteration of protein glycosylation in liver diseases. J. Hepatol. 2009, 50, 592-603. [CrossRef]

20. Zühlsdorf, A.; Park, J.H.; Wada, Y.; Rust, S.; Reunert, J.; Duchesne, I.; Grüneberg, M.; Marquardt, T. Transferrin variants: Pitfalls in the diagnostics of Congenital disorders of glycosylation. Clin. Biochem. 2015, 48, 11-13. [CrossRef]

21. Miyatake, M.; Kuno, T.; Kita, A.; Katsura, K.; Takegawa, K.; Uno, S.; Nabata, T.; Sugiura, R. Valproic Acid Affects Membrane Trafficking and Cell-Wall Integrity in Fission Yeast. Genetics 2007, 175, 1695-1705. [CrossRef]

22. Iori, V.; Maroso, M.; Rizzi, M.; Iyer, A.M.; Vertemara, R.; Carli, M.; Agresti, A.; Antonelli, A.; Bianchi, M.E.; Aronica, E.; et al. Receptor for Advanced Glycation Endproducts is upregulated in temporal lobe epilepsy and contributes to experimental seizures. Neurobiol. Dis. 2013, 58, 102-114. [CrossRef]

23. Xie, J.; Méndez, J.D.; Méndez-Valenzuela, V.; Hernandez, M.M.A. Cellular signalling of the receptor for advanced glycation end products (RAGE). Cell. Signal. 2013, 25, 2185-2197. [CrossRef] [PubMed]

24. Yuen, A.W.; Bell, G.S.; Peacock, J.L.; Koepp, M.M.; Patsalos, P.N.; Sander, J.W. Effects of AEDs on biomarkers in people with epilepsy: CRP, HbA1c and eGFR. Epilepsy Res. 2010, 91, 187-192. [CrossRef] [PubMed]

25. Guo, M.; Wang, J.; Qi, H.; Liu, F.; Yao, L.; Zhang, S.; Li, K. Polymorphisms in the receptor for advanced glycation end products gene are associated with susceptibility to drug-resistant epilepsy. Neurosci. Lett. 2016, 619, 137-141. [CrossRef] [PubMed] 